\title{
Astaxanthin Prevents Decreases in Superoxide Dismutase 2 Level and Superoxide Dismutase Activity in Helicobacter pylori-infected Gastric Epithelial Cells
}

SHORT

COMMUNICATION

\author{
Suhn Hyung Kim, Joo Weon Lim, Hyeyoung Kim \\ Department of Food and Nutrition, Brian Korea 21 PLUS Project, College of Human Ecology, Yonsei University, Seoul, Korea
}

\begin{abstract}
Background: Helicobacter pylori increases production of reactive oxygen species (ROS), which activates inflammatory and carcinogenesis-related signaling pathways in gastric epithelial cells. Therefore, reducing ROS, by upregulating antioxidant enzyme, such as superoxide dismutase (SOD), may be a novel strategy to prevent $H$. pylori-associated gastric diseases. Astaxanthin is an antioxidant carotenoid that prevents oxidative stress-induced cell injury. The present study was aimed to determine whether $H$. pylori decreases SOD activity by changing the levels of SOD1/SOD2 and whether astaxanthin prevents changes in SOD levels and activity in $\mathrm{H}$. pylori-infected gastric epithelial AGS cells.

Methods: AGS cells were pre-treated with astaxanthin for 3 hours prior to $H$. pylori infection and cultured for 1 hour in the presence of $H$. pylori. SOD levels and activity were assessed by Western blot analysis and a commercial assay kit, respectively. Mitochondrial ROS was determined using MitoSOX fluorescence.

Results: H. pylori decreased SOD activity and the SOD2 level, but increased mitochondrial ROS in AGS cells. The SOD1 level was not changed by $H$. pylori infection. Astaxanthin prevented $H$. pylori-induced decreases in the SOD2 level and SOD activity and reduced mitochondrial ROS in AGS cells.
\end{abstract}

Conclusions: Consumption of astaxanthin-rich food may prevent the development of $\mathrm{H}$. pylori-associated gastric disorders by suppressing mitochondrial oxidative stress.

(J Cancer Prev 2019;24:54-58)

Key Words: Astaxanthin, Helicobacter pylori, Gastric diseases, Reactive oxygen species, Superoxide dismutase

\section{INTRODUCTION}

Helicobacter pylori infection increases oxidative stress in the infected cells, which contributes to the development of gastric diseases including gastric cancer [1-4]. Mitochondria are the center of energy production and generate reactive oxygen species (ROS) from electron transport respiratory chain [5]. Our recent study has demonstrated that interluekin-8 expression is highly related to mitochondrial ROS in gastric epithelial cells infected with $H$. pylori [6].

Superoxide dismutases (SODs) are important antioxidant enzymes, which catalyze superoxide anion dismutation on hydrogen peroxide and oxygen. Therefore, expression and activation of SODs have great impact on cellular oxidative response. SODs have three isoforms; cytosolic SOD (SOD1, Cu/Zn SOD), mitochondrial SOD (SOD2, Mn SOD) and extracellular SOD (SOD3, ecSOD) [7]. Because ROS activate signaling pathways related to inflammation, immune system, and cancer development, regulating ROS levels in the cells is important for maintaining normal physiology of the cells [8]. Therefore, it is essential to investigate whether $H$. pyloriaffects SOD1/SOD2 levels and SOD activity in gastric epithelial cells to better understand pathologic mechanisms of $H$. pylori-induced gastric diseases. Because SOD3 is a secretory extracellular $\mathrm{Cu} / \mathrm{Zn}$-containing SOD and primarily

Received March 13, 2019, Revised March 22, 2019, Accepted March 22, 2019

Correspondence to: Hyeyoung Kim

E-mail: kim626@yonsei.ac.kr, ORCID: Hyeyoung Kim, https://orcid.org/0000-0002-7019-917X

Copyright (C) 2019 Korean Society of Cancer Prevention

(c) This is an Open Access article distributed under the terms of the Creative Commons Attribution Non-Commercial License (http://creativecommons.org/licenses/by-nc/4.0) which permits unrestricted non-commercial use, distribution, and reproduction in any medium, provided the original work is properly cited. 
located in the extracellular matrix and extracellular fluids [9], we determine the changes in SOD1 and SOD2 levels in the present study.

Astaxanthin, a potent antioxidant xanthophyll, contributes to red-orange color of aquatic animals including shrimp, salmon, and lobster [10]. We previously demonstrated that astaxanthin acted as a PPAR- $\gamma$ agonist and increased the catalase level through PPAR- $\gamma$ activation in gastric epithelial cells infected with $H$. pylori [6]. Some evidence showed that SODs are PPAR- $\gamma$ target genes and SOD activity was positively correlated with PPAR $\gamma$ activity in aorta and cardiomyocytes [11,12]. Therefore, we hypothesize that astaxanthin may decrease ROS levels by increasing SOD activity in gastric epithelial cells infected with $H$. pylori.

The present study is aimed to determine whether $H$. pylori changes the levels of SOD1 and SOD2 and SOD activity, and whether $H$. pylori-induced changes in SOD levels and activity are inhibited by astaxanthin in gastric epithelial AGS cells

\section{MATERIALS AND METHODS}

\section{Reagents}

Astaxanthin (Sigma-Aldrich, St. Louis, MO, USA), dissolved in dimethyl sulfoxide and stored under nitrogen gas at $-80^{\circ} \mathrm{C}$, was thawed and added to fetal bovine serum to achieve the desired concentrations.

\section{Cell line and culture condition}

The human gastric epithelial AGS cells (gastric adenocarcinoma, ATCC CRL 1739; American Type Culture Collection, Rockville, MD, USA) were grown in RPMI 1640 medium (GIBCO, Grand Island, NY, USA) supplemented with 10\% FBS, 2 mM glutamine, $100 \mathrm{U} / \mathrm{mL}$ penicillin, and $100 \mu \mathrm{g} / \mathrm{mL}$ streptomycin (Sigma-Aldrich) and cultured at $37^{\circ} \mathrm{C}$ under $95 \%$ air and $5 \% \mathrm{CO}_{2}$.

H. pylori bacteria (NCTC 11637; American Type Culture Collection) were grown on chocolate agar plates (Becton Dickinson Microbiology Systems, Cockeysvile, MD, USA) at $37^{\circ} \mathrm{C}$, under microaerophilic conditions, using an anaerobic chamber (BBL Campy Pouch ${ }^{\circledR}$ System; Becton Dickinson Microbiology Systems, Franklin Lakes, NJ, USA).

AGS cells were seeded overnight to reach $80 \%$ confluency. The H. pylori was harvested and suspended in antibiotic-free RPMI 1640 medium supplemented with $10 \% \mathrm{FBS}$, and then added to the AGS cell culture at the ratio of bacterium: AGS cells, $50: 1$. AGS cells $\left(1.0 \times 10^{5} / \mathrm{mL}\right.$ ) were pre-treated with astaxanthin (at final concentration of 1 and $5 \mu \mathrm{M}$ ) for 3 hours before adding the $H$. pylori and cultured for 1 hour.
3. Preparation of cell extracts and Western blot analysis for superoxide dismutase 1 and superoxide dismutase 2

Preparation of cell extracts and mitochondrial ROS levels were determined by the method described previously [10]. Western blotting was performed by the method previously described $[13,14]$. Briefly, whole-cell extracts were loaded onto $8 \%$ to $10 \%$ SDS PAGE (6-40 $\mu$ g protein/lane) and separated by electrophoresis under reducing conditions. The proteins were verified using reversible staining with Ponceau S. The membranes were blocked using 3\% non-fat dry milk in TBS and 0.2\% Tween 20 (TBS-T). The proteins were detected using antibodies for SOD1 (sc-11407; Santa Cruz Biotechnology, Dallas, TX, USA), SOD2 (sc-30080; Santa Cruz Biotechnology), and actin as a loading control (sc-1615; Santa Cruz Biotechnology) in TBS-T solution containing 3\% dry milk, and incubated overnight at $4^{\circ} \mathrm{C}$. After washing with TBS-T, the primary antibodies were detected using horseradish peroxidase-conjugated secondary antibodies (anti-mouse, antirabbit, anti-goat), and visualized using the enhanced chemiluminescence detection system (Santa Cruz Biotechnology). Protein levels of SOD1 and SOD2 were compared to that of actin and expressed as the percentage density ratio.

\section{Determination of mitochondrial reactive oxygen} species levels and superoxide dismutase activity

Mitochondrial ROS levels were determined by the method described previously [15]. SOD activity level was measured using SOD assay kit (19160-1KT-F; Sigma-Aldrich). SOD activity was defined in unit/mg protein.

\section{Statistical analysis}

Statistical analysis was performed using one-way ANOVA, followed by Newman-Keul's tests. The results were expressed as the mean $\pm \mathrm{SE}$ of three different experiments. A $P$-value of 0.05 or less was considered statistically significant.

\section{RESULTS}

As shown in Figure 1A, AGS cells with H. pylori significantly increased the levels of mitochondrial ROS (column "None" vs. column "Control"). Astaxanthin $(5 \mu \mathrm{M})$ reduced mitochondrial ROS levels in $H$. pylori-stimulated AGS cells (column "AST" vs. column "Control"). Figure 1B showed that $H$. pylori reduced the protein level of SOD2, but not the SOD1 level in AGS cells (column "None" vs. column "Control"). Astaxanthin dose-dependently 


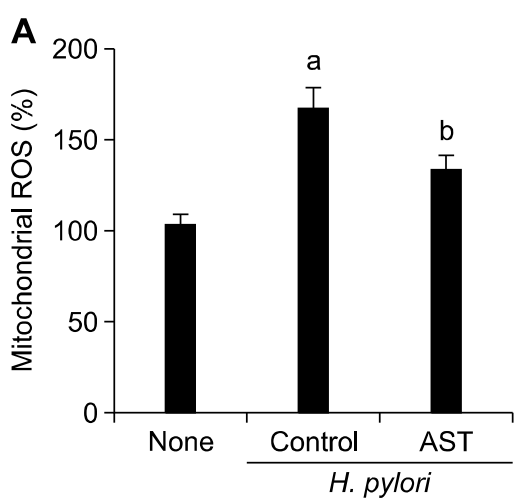

B
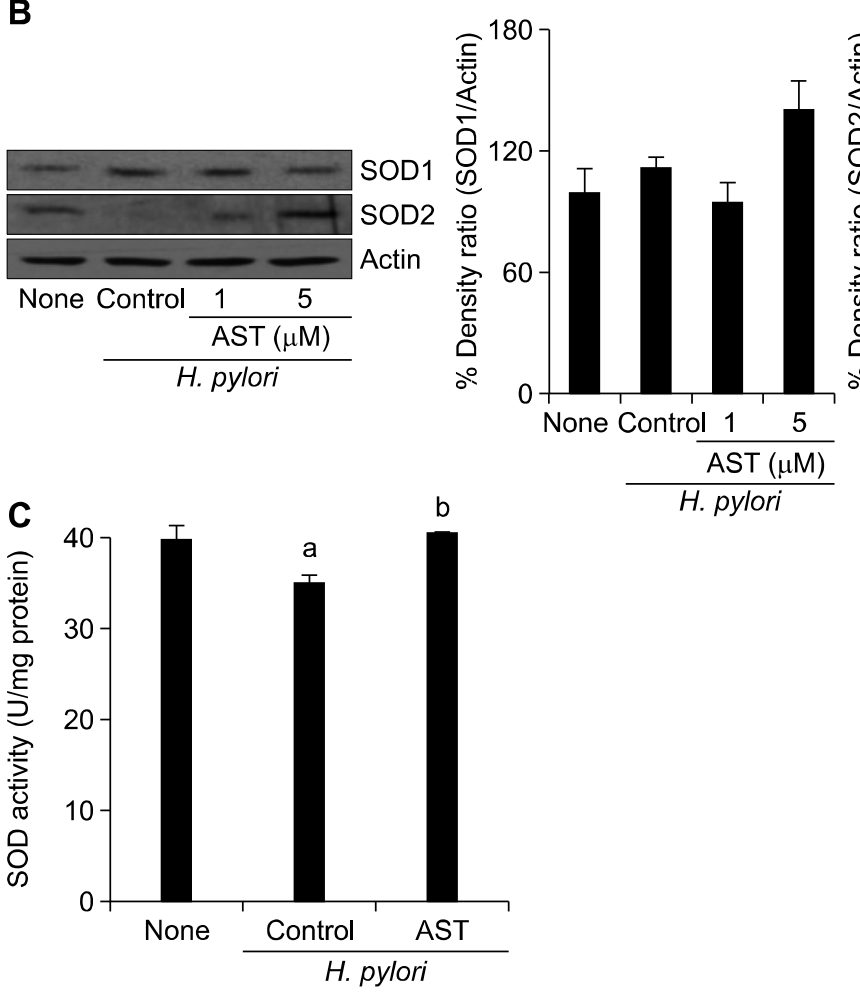

prevented loss of SOD2 level in H. pylori-infected cells (column "AST 1" vs. column "Control") (column "AST 5" vs. column "Control"). Astaxanthin ( $5 \mu \mathrm{M})$ tended to increase the SOD1 level in $H$. pylori-infected cells. However, there are no significant differences among experimental groups. SOD activity in AGS cells decreased by $H$. pylori infection (Fig. 1C). Astaxanthin ( $5 \mu \mathrm{M}$ ) prevented a decrease in SOD activity in $H$. pylori-infected cells (column "AST" vs. column "Control").

The results demonstrate that $H$. pylori infection reduces the SOD2 level and SOD activity, which may increase mitochondrial ROS in AGS cells. H. pylori-induced oxidative stress may be caused by decreased mitochondrial antioxidant capacity in the infected cells. Astaxanthin inhibits $H$, pylori-induced mitochondrial SOD loss and mitochondrial oxidative stress in the infected cells.
Figure 1. Effect of astaxanthin (AST) on mitochondrial reactive oxygen species (ROS), superoxide dismutase (SOD) levels and SOD activity in Helicobacter pylori-infected AGS cells. The cells were pre-treated with 1 or $5 \mu \mathrm{M}$ of AST for 3 hours and then stimulated with $H$. pylori for 1 hour. (A) Mitochondrial ROS levels were measured by MitoSOX fluorescence. "None" corresponds to uninfected cells; "Control" corresponds to the cells infected with $H$. pylori alone; "AST" corresponds to the cells infected with $H$. pylori with treatment of $5 \mu \mathrm{M}$ AST. ${ }^{a} P<0.05$ vs. "none"; ${ }^{\mathrm{b}} P<0.05$ vs. "control". (B) The levels of SOD1 and SOD2 were measured by Western blot analysis for uninfected AGS cells (none), those infected with $H$. pylori (control), AGS cells infected with $H$. pylori and treated with 1 or $5 \mu \mathrm{M}$ AST (left panel). The protein level of SOD1 and SOD2 were compared to that of actin and expressed as the percentage density ratio. All values were expressed as mean \pm SE of three different experiments. ${ }^{a} P<0.05$ vs. "none"; ${ }^{\mathrm{b}} P<0.05$ vs. "control" (right panel). (C) SOD activity was measured by a commercial assay kit. The description of the columns is the same as in (A). ${ }^{\mathrm{a}} P<0.05$ vs. "none"; ${ }^{\mathrm{b}} P<0.05$ vs. "control".

\section{DISCUSSION}

In the present study, decreased SOD2 levels and total SOD activity were found in $H$. pylori-infected gastric epithelial cells. Therefore, loss of SOD2 may increase mitochondrial ROS in $H$. pylori-infected gastric epithelial cells. Interestingly, the level of cytosolic SOD1 was not changed by $H$. pylori infection. Smoot et al. [16] demonstrated that $H$. pylori had no effect on SOD1 levels in AGS cells at 24 hour-culture. However, they showed that $H$. pylori increased SOD2 activity and catalase activity in AGS cells. In that study, AGS cells were cultured in the medium containing $1 \times 10^{7}$ bacteria/mL for 24 hours. In the present study, we used the ratio of bacterium: AGS cells $\left(1.0 \times 10^{5} / \mathrm{mL}\right), 50: 1$. Therefore, they used the higher bacterial infection ratio and cultured 24 
hours. Since we used 1 hour-culture, different infection ratio and culture time may cause different result on SOD2 activity induced by $H$. pylori infection.

We previously demonstrated that astaxanthin activated PPAR- $\gamma$ to induce catalase expression in $H$. pylori-infected gastric epithelial cells [6]. PPAR- $\gamma$ has transcriptional activity towards a number of genes involved in metabolic processes as well as antioxidant genes $[17,18]$. PPAR- $\gamma$ increases expression of catalase [19] and SOD1 expression is dependent on PPAR- $\gamma$ activation [11]. In the present study, the SOD2 level increased by astaxanthin treatment in $H$. pylori-infected cells. SOD1 level was tended to be increased by a high concentration of astaxanthin even though there was no significant difference between groups. For the further study, various concentrations of astaxanthin and various infection rate should be used to assess inhibitory effect of astaxanthin on decreases in SOD levels in $H$. pylori-infected cells. Since we only used one cell line in the present study, it will be necessary to use more gastric epithelial cell lines to confirm the effect of astaxanthin for the further study.

Regarding the studies of astaxanthin on mitochondrial function, astaxanthin prevented loss of mitochondrial respiration in cardiac and neuronal damage [20-23]. These studies suggest the possible protective effect of astaxanthin against mitochondrial malfunction.

Here we found that $H$. pylori decreased SOD activity and the SOD2 level, but increased mitochondrial ROS in AGS cells. the SOD1 level was not changed by $H$. pylori infection. Astaxanthin prevented $H$. pylori-induced decreases in SOD2 level and SOD activity and reduced mitochondrial ROS in AGS cells. Therefore, consumption of astaxanthin-rich food may prevent the development of $H$. pylori-associated gastric disorders by suppressing mitochondrial oxidative stress.

\section{ACKNOWLEDGMENTS}

This study was supported by a grant from the National Research Foundation (NRF) of Korea, which is funded by the Korean Government (NRF-2018R1A2B2005575).

\section{CONFLICTS OF INTEREST}

No potential conflicts of interest were disclosed.

\section{REFERENCES}

1. Piao JY, Lee HG, Kim SJ, Kim DH, Han HJ, Ngo HK, et al. Helicobacter pylori activates IL-6-STAT3 signaling in human gas- tric cancer cells: potential roles for reactive oxygen species. Helicobacter 2016;21:405-16.

2. Butcher LD, den Hartog G, Ernst PB, Crowe SE. Oxidative stress resulting from Helicobacter pylori infection contributes to gastric carcinogenesis. Cell Mol Gastroenterol Hepatol 2017;3:316-22.

3. Gobert AP, Wilson KT. Human and Helicobacter pylori interactions determine the outcome of gastric diseases. Curr Top Microbiol Immunol 2017; 400:27-52.

4. Gobert AP, Wilson KT. Polyamine- and NADPH-dependent generation of ROS during Helicobacter pylori infection: a blessing in disguise. Free Radic Biol Med 2017;105:16-27.

5. Dan Dunn J, Alvarez LA, Zhang X, Soldati T. Reactive oxygen species and mitochondria: a nexus of cellular homeostasis. Redox Biol 2015;6:472-85.

6. Kim SH, Lim JW, Kim H. Astaxanthin inhibits mitochondrial dysfunction and interleukin-8 expression in Helicobacter pylori-infected gastric epithelial cells. Nutrients 2018;10:E1320.

7. Miller AF. Superoxide dismutases: ancient enzymes and new insights. FEBS Lett 2012;586:585-95.

8. Wang Y, Branicky R, Noë A, Hekimi S. Superoxide dismutases: dual roles in controlling ROS damage and regulating ROS signaling. J Cell Biol 2018;217:1915-28.

9. Fukai T, Ushio-Fukai M. Superoxide dismutases: role in redox signaling, vascular function, and diseases. Antioxid Redox Signal 2011;15:1583-606.

10. Ambati RR, Phang SM, Ravi S, Aswathanarayana RG. Astaxanthin: sources, extraction, stability, biological activities and its commercial applications: a review. Mar Drugs 2014;12:128-52.

11. Umeji K, Umemoto S, Itoh S, Tanaka M, Kawahara S, Fukai T, et al. Comparative effects of pitavastatin and probucol on oxidative stress, $\mathrm{Cu} / \mathrm{Zn}$ superoxide dismutase, PPAR-gamma, and aortic stiffness in hypercholesterolemia. Am J Physiol Heart Circ Physiol 2006;291:H2522-32.

12. Ding G, Fu M, Qin Q, Lewis W, Kim HW, Fukai T, et al. Cardiac peroxisome proliferator-activated receptor gamma is essential in protecting cardiomyocytes from oxidative damage. Cardiovasc Res 2007;76:269-79.

13. Park B, Lim JW, Kim H. Lycopene treatment inhibits activation of Jak1/Stat3 and Wnt/ $\beta$-catenin signaling and attenuates hyperproliferation in gastric epithelial cells [published online ahead of print July 21, 2018]. Nutr Res. doi: 10.1016/j.nutres.2018.07.010.

14. Cha B, Lim JW, Kim KH, Kim H. 15-deoxy-D12,14-prostaglandin J2 suppresses RANTES expression by inhibiting NADPH oxidase activation in Helicobacter pylori-infected gastric epithelial cells. J Physiol Pharmacol 2011;62:167-74.

15. Hwang S, Lim JW, Kim H. Inhibitory effect of lycopene on amyloid- $\beta$-induced apoptosis in neuronal cells. Nutrients 2017; 9:E883.

16. Smoot DT, Elliott TB, Verspaget HW, Jones D, Allen CR, Vernon $\mathrm{KG}$, et al. Influence of Helicobacter pylori on reactive oxygen-induced gastric epithelial cell injury. Carcinogenesis 2000; 21:2091-5.

17. Semple RK, Chatterjee VK, O'Rahilly S. PPAR gamma and human metabolic disease. J Clin Invest 2006;116:581-9.

18. Dovinová I, Barancik M, Majzunova M, Zorad S, Gajdosechová L, Gresová L, et al. Effects of PPAR $\gamma$ agonist pioglitazone on redox-sensitive cellular signaling in young spontaneously hypertensive rats. PPAR Res 2013;2013:541871.

19. Girnun GD, Domann FE, Moore SA, Robbins ME. Identification 
of a functional peroxisome proliferator-activated receptor response element in the rat catalase promoter. Mol Endocrinol 2002;16:2793-801.

20. Fan CD, Sun JY, Fu XT, Hou YJ, Li Y, Yang MF, et al. Astaxanthin attenuates homocysteine-induced cardiotoxicity in vitro and in vivo by inhibiting mitochondrial dysfunction and oxidative damage. Front Physiol 2017;8:1041.

21. Pongkan W, Takatori O, Ni Y, Xu L, Nagata N, Chattipakorn SC, et al. $\beta$-Cryptoxanthin exerts greater cardioprotective effects on cardiac ischemia-reperfusion injury than astaxanthin by attenu- ating mitochondrial dysfunction in mice. Mol Nutr Food Res 2017. doi: 10.1002/mnfr.201601077.

22. Liu X, Shibata T, Hisaka S, Osawa T. Astaxanthin inhibits reactive oxygen species-mediated cellular toxicity in dopaminergic SH-SY5Y cells via mitochondria-targeted protective mechanism. Brain Res 2009;1254:18-27.

23. Lee DH, Kim CS, Lee YJ. Astaxanthin protects against MPTP/MPP+-induced mitochondrial dysfunction and ROS production in vivo and in vitro. Food Chem Toxicol 2011;49:271-80. 